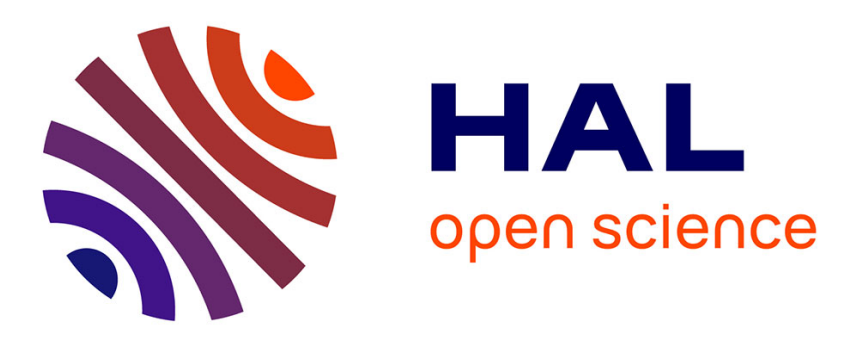

\title{
Credit Risk with asymmetric information on the default threshold
}

\author{
Caroline Hillairet, Ying Jiao
}

\section{To cite this version:}

Caroline Hillairet, Ying Jiao. Credit Risk with asymmetric information on the default threshold. Stochastics: An International Journal of Probability and Stochastic Processes, 2012, 84 (2-3), pp.183198. 10.1080/17442508.2011.575944 . hal-00663136

\section{HAL Id: hal-00663136 https://hal.science/hal-00663136}

Submitted on 26 Jan 2012

HAL is a multi-disciplinary open access archive for the deposit and dissemination of scientific research documents, whether they are published or not. The documents may come from teaching and research institutions in France or abroad, or from public or private research centers.
L'archive ouverte pluridisciplinaire HAL, est destinée au dépôt et à la diffusion de documents scientifiques de niveau recherche, publiés ou non, émanant des établissements d'enseignement et de recherche français ou étrangers, des laboratoires publics ou privés. 


\title{
Credit Risk with asymmetric information on the default threshold*
}

\author{
CARoline HiLlaireT ${ }^{\dagger}$ Ying JiAO $\ddagger$ \\ International Conference on Stochastic Analysis and Applications, \\ Hammamet - October 12-17, 2009
}

\begin{abstract}
We study the impact of asymmetric information in a general credit model where the default is triggered when a fundamental diffusion process of the firm passes below a random threshold. Inspired by some recent technical default events during the financial crisis, we consider the role of the firm's managers who choose the level of the default threshold and have complete information. However, other investors on the market only have partial observations either on the process or on the threshold. We specify the accessible information for different types of investors. Besides the framework of progressive enlargement of filtrations usually adopted in the credit risk modelling, we also combine the results on initial enlargement of filtrations to deal with the uncertainty on the default threshold. We consider several types of investors who have different information levels and we compute the default probabilities in each case. Numerical illustrations show that the insiders who have extra information on the default threshold obtain better estimations of the default probability compared to the standard market investors.
\end{abstract}

\section{Introduction}

In the credit risk analysis, it is crucial to model the default event and to forecast the default probabilities for pricing and risk management purposes. In the literature, there exist two main modelling approaches: the structural one and the reduced-form one. The structural approach provides a convincing economic interpretation, where the original

${ }^{*}$ This research is part of the Chair Financial Risks of the Risk Foundation, the Chair Derivatives of the Future sponsored by the Fédération Bancaire Française, and the Chair Finance and Sustainable Development sponsored by EDF and Calyon.

${ }^{\dagger}$ CMAP Ecole Polytechnique, caroline.hillairet@polytechnique.edu

${ }^{\ddagger}$ LPMA Université Paris 7, jiao@math.jussieu.fr 
idea goes back to the paper of Merton [20]. The default is triggered when a fundamental process $X$ of the firm passes below a deterministic threshold $L$. The fundamental process may represent the asset value or the total cash flow. The level $L$ is chosen by the firm's managers according to some economic criterions, for example, maximizing the equity value. The default time defined in the structural approach is a predictable stopping time and is considered as an observable event once the process approaches the threshold. In the reduced-form approach, the default is assumed to arrive in a more "surprising" way, especially in the short term. The uncertainty is often characterized by the level of the credit spread or the default intensity. The model parameters can be calibrated from market data.

The links between the two approaches have been well studied by many authors. There are in general two methods to introduce short-term default risks in a classical structural model. The first way is to consider a generalized first-passage model where the default threshold $L$ is supposed to be random (e.g. [7, 18, 10]). The second way is to suppose that the process $X$ is partially observed by the investors (e.g. $[6,4,16,12,3,2])$. In both cases, the information accessibility plays an important role.

Let us consider the first-passage model in a general setting. Let $(\Omega, \mathcal{A}, \mathbb{P})$ be a probability space where $\mathcal{A}$ is a $\sigma$-algebra of $\Omega$ representing the total information on the market. We consider a firm and model its default time as the first time that a continuous time process $\left(X_{t}\right)_{t \geq 0}$ reaches some default barrier $L$, i.e.,

$$
\tau=\inf \left\{t: X_{t} \leq L\right\} \quad \text { where } X_{0}>L
$$

with the convention that $\inf \emptyset=+\infty$. Denote by $\mathbb{F}=\left(\mathcal{F}_{t}\right)_{t \geq 0}$ the filtration generated by the process $X$, i.e., $\mathcal{F}_{t}=\sigma\left(X_{s}, s \leq t\right) \vee \mathcal{N}$ satisfying the usual conditions where $\mathcal{N}$ denotes the $\mathbb{P}$ null sets. We introduce the decreasing process $X^{*}$ defined as $X_{t}^{*}=\inf \left\{X_{s}, s \leq t\right\}$. Then (1.1) can be rewritten as

$$
\tau=\inf \left\{t: X_{t}^{*}=L\right\}
$$

Note that the information of $X_{t}^{*}$ is contained in the $\sigma$-algebra $\mathcal{F}_{t}$.

Such construction of a default time adapts to both structural and reduced form approaches of the default modelling. In the structural approach models, $L$ is a constant or a deterministic function $L(t)$, then $\tau$ defined in (1.1) is a predictable $\mathbb{F}$-stopping time (since the firm value $\left(X_{t}\right)_{t \geq 0}$ is a continuous time process). In the reduced-form approach ${ }^{1}$, the default barrier $L$ is unknown and is described as a random variable in $\mathcal{A}$. In the widely used Cox Model [18], the barrier $L$ is supposed to be independent of $\mathcal{F}_{\infty}$ and the law of $L$ is known. In the incomplete information models such as $[6,16,12]$, the whole process $X$ can not be fully observed, so the information concerning $X$ is represented by some

\footnotetext{
${ }^{1}$ In the classical reduced-form model such as the Cox-process model in Lando [18], $X$ is an increasing process - the compensator process of default - instead of a decreasing one, and $L$ is an upper bound. See $\S 3.2$ for details.
} 
subfiltration of $\mathbb{F}$. In all the models, the default probabilities are computed with respect to the observable information.

In this work, we are specially interested in the information asymmetry on the default threshold $L$. This is motivated by some recent "technical default events" during the crisis: the firm is still in a relatively healthy situation, nevertheless, the managers have decided to close the activities and the default occurs. Hence, the default barrier in this case is a random variable whose value is chosen by the managers. In the literature, the information on the value process of the firm has been thoroughly studied. However, only few works concentrate on the default threshold. Giesecke and his co-author consider in [9] and [10] the case where the process $X$ is perfectly observed, but where $L$ is a random variable that is not observed. That is, they consider the filtration generated by $X$ and by $L$ so that the default time is a stopping time. Our approach is different: we study the problem by using the theory of enlargement of filtrations. We consider the default threshold to be an exogenous source of risk and we add the knowledge on $L$ to the whole information flow. The information of the managers becomes then $\mathbb{F} \vee \sigma(L)$. In addition, we consider another type of investors, the insiders, who do not have the full access to the threshold value but know some extra information on it compared to other market investors. We compute conditional default probabilities for these different investors and we show the importance of the information level for their estimations of default probabilities.

The rest of this paper is organized as follows. In Section 2, we introduce different information structures for various agents on the market. We distinguish the role of the managers who choose the default barrier $L$, the insiders who have information on $L$ and the investors who only observe the occurrence of the default. We precise the mathematical hypotheses, using the language of enlargement of filtrations. Section 3 is devoted to the explicit calculations on the conditional default probabilities. We then give numerical illustrations in Section 4 to quantify how different partial information impact the estimations of the default probabilities.

\section{The informational structure}

On the financial market, the available information for each agent is various. On one hand, there is a strong information asymmetry between the managers and the investors of the firm. The important point is that the managers have prior information on whether the firm will default and the timing of the default. On the other hand, market investors do have different information. We now describe the different information concerning the firm and the threshold : we will consider four levels of information on the default threshold $L$ and the underlying process $X$. In the following, we suppose that the default barrier is fixed at the initial date by the manager as the realization of the random variable $L$, and that all investors observe the occurrence of the default. 


\section{$2.1 \quad$ Full information}

The managers have perfect information on the firm. At any time $t$, they know the continuous firm value, together with the default barrier. In other words, the managers have complete information on both $X$ and $L$. The information of the managers - called the full information - is then given at time $t \geq 0$ by

$$
\mathcal{G}_{t}^{M}:=\mathcal{F}_{t} \vee \sigma(L)
$$

The filtration $\mathbb{G}^{M}=\left(\mathcal{G}_{t}^{M}\right)_{t \geq 0}$ is in fact the initial enlargement of the filtration $\mathbb{F}$ with the random variable $L$. For the manager, $\tau$ is a predictable $\mathbb{G}^{M}$-stopping time. Let us precise better the nature of this initial information.

Assumption 2.1 We assume that $L$ is an $\mathcal{A}$-measurable random variable with values in $\mathbb{R}$ which satisfies the assumption: $\mathbb{P}\left(L \in \cdot \mid \mathcal{F}_{t}\right)(\omega) \sim \mathbb{P}(L \in \cdot)$ for all $t$ for $\mathbb{P}$ almost all $\omega \in \Omega$.

Remark: Assumption 2.1 is satisfied if $L$ is independent of $\mathcal{F}_{\infty}$.

Assumption 2.1 is the standard assumption by Jacod [14, 15]. We denote by $P_{t}^{L}(\omega, d x)$ a regular version of the conditional law of $L$ given $\mathcal{F}_{t}$ and by $P^{L}$ the law of $L$. According to Jacod, there exists a measurable version of the conditional density $p_{t}(x)(\omega)=\frac{d P_{t}^{L}}{d P^{L}}(\omega, x)$ which is a $(\mathbb{F}, \mathbb{P})$-martingale and for all $t \geq 0, p_{t}(L)>0 \quad \mathbb{P}$ almost surely.

Grorud et al. [11] proved that Assumption 2.1 is equivalent to the existence of a probability measure equivalent to $\mathbb{P}$ and under which for any $t \geq 0, \mathcal{F}_{t}$ and $\sigma(L)$ are independent. We consider the only one, denoted by $\mathbb{Q}^{L}$, which is identical to $\mathbb{P}$ on $\mathcal{F}_{\infty}$. The probability measure $\mathbb{Q}^{L}$ is characterized by the density process

$$
E_{\mathbb{Q}^{L}}\left[\frac{d \mathbb{P}}{d \mathbb{Q}^{L}} \mid \mathcal{G}_{t}^{M}\right]=p_{t}(L) .
$$

It will play a key role in the computation of the conditional default probability.

\subsection{Progressive information}

The progressive information is the information level of an ordinary investor who observes the process $X$ but does not have any knowledge on the barrier $L$, except that he observes at time $t$ whether the default has occurred up to $t$ and if so, the exact timing of default. His information is given as the progressive enlargement of filtration of $\mathbb{F}$ with $\tau$ :

$$
\mathbb{G}=\left(\mathcal{G}_{t}\right)_{t \geq 0} \text { with } \mathcal{G}_{t}=\mathcal{F}_{t} \vee \mathcal{D}_{t}
$$

where $\mathbb{D}=\left(\mathcal{D}_{t}=\cup_{s>t} \sigma(\tau \wedge s)\right)_{t \geq 0}$ is the minimal right-continuous filtration which makes $\tau$ a $\mathbb{D}$-stopping time. The filtration $\mathbb{G}=\left(\mathcal{G}_{t}\right)_{t \geq 0}$ corresponds to the standard information 
flow in the credit modelling. We call this information the "progressive information" on $L$. We see that the filtration $\mathbb{G}^{M}$ is larger than $\mathbb{G}$.

Remark: If $L$ is independent of $\mathcal{F}_{\infty}$, then the so-called (H)-hypothesis is satisfied: every $(\mathbb{F}, \mathbb{P})$ local martingale is also a $(\mathbb{G}, \mathbb{P})$ local martingale. The $(\mathrm{H})$-hypothesis is equivalent to the equality $\mathbb{P}\left(\tau>t \mid \mathcal{F}_{t}\right)=\mathbb{P}\left(\tau>t \mid \mathcal{F}_{\infty}\right)$ for all $t \geq 0$. This hypothesis is standard in the credit risk modelling. For example, the widely used Cox process model [18] satisfies this hypothesis.

\subsection{Noisy full information}

We now consider an intermediary case: the case of an insider who is an investor having additional observations on $L$ besides the information on $\mathbb{D}$ and on $\mathbb{F}$. We assume that the additional information on the barrier $L$ changes through time : the knowledge on $L$ is distorted by an independent noise, and is getting to him clearer as time evolves. More precisely, we suppose that this insider observes $L_{s}=L+\epsilon_{s}$ at time $s$ with $\left(\epsilon_{s}\right)_{s \geq 0}$ being an independent noise perturbing the information on $L$. The information of the insider is then given by the following filtration $\mathbb{G}^{I}=\left(\mathcal{G}_{t}^{I}\right)_{t \geq 0}$ and is denoted as the "noisy full information":

\section{Assumption 2.2}

For any $t \geq 0, \mathcal{G}_{t}^{I}=\mathcal{F}_{t}^{I} \vee \mathcal{D}_{t}$ where $\mathcal{F}_{t}^{I}=\cap_{u>t}\left(\mathcal{F}_{u} \vee \sigma\left(L_{s}, s \leq u\right)\right), L_{s}=L+\epsilon_{s}$ with

- $\epsilon=\left\{\epsilon_{t}, t \geq 0\right\}$ is independent of $\mathcal{F}_{\infty} \vee \sigma(L)$.

- $\mathbb{P}\left(L \in \cdot \mid \mathcal{F}_{t}\right)(\omega) \sim \mathbb{P}(L \in \cdot)$ for all $t$ for $\mathbb{P}$ almost all $\omega \in \Omega$.

If we work on a finite horizon $T$, the last two assumptions are

- $\epsilon=\left\{\epsilon_{t}, t \leq T\right\}$ is independent of $\mathcal{F}_{T} \vee \sigma(L)$,

- $\mathbb{P}\left(L \in \cdot \mid \mathcal{F}_{t}\right)(\omega) \sim \mathbb{P}(L \in \cdot)$ for all $t \in[0, T[$ for $\mathbb{P}$ almost all $\omega \in \Omega$.

The process $\epsilon$ represents an additional noise that perturbs the knowledge of the barrier $L$. Therefore one expects in general that the variance of the noise decreases to zero as time $t$ goes to infinity.

\subsection{Delayed information}

In this subsection, we consider the case where the process $X$ driving the default risk is not totally observable for all agents. We suppose that at date 0, all investors are completely informed on the firm value. Later on, they will be differently informed on the process $X$. Let us assume in the sequel that the process $X$ is associated with a standard Brownian motion $B$ (for example, $X$ is a geometric Brownian motion or the solution of some SDE). Let $\mathcal{N}$ denotes the $\mathbb{P}$ null sets and we assume that $\mathcal{F}_{t}=\sigma\left(B_{s}, s \leq t\right) \vee \mathcal{N}$ where $\mathbb{F}=\left(\mathcal{F}_{t}\right)_{t \geq 0}$ represents the information of an investor having complete information of the fundamental process $X$. Most investors on the market only have an incomplete observation described 
by an auxiliary filtration of $\mathbb{F}$. In the literature, there are several ways to describe the incomplete information:

Example 2.3 (Noisy information) A structural type model with deterministic barrier is studied in [3]. The partial information is represented by an auxiliary process $\beta$ depending on some noisy signal of the process $X$. The information of an investor observing the noisy signal of $X$ is represented by the filtration $\mathcal{F}_{t}^{\beta}:=\sigma\left(\beta_{s}, s \leq t\right) \vee \mathcal{N}$.

Example 2.4 (Delayed information) The investors may have a delayed (continuous or discrete) observation of the fundamental process $X$, this type of models have been considered, among others, by $[6,4,16,12]$. In this case, the observable information is characterized by a sub-filtration $\mathbb{F}^{D}=\left(\mathcal{F}_{t}^{D}\right)_{t \geq 0}$ of $\mathbb{F}$, constructed by either a time change (continuously delayed filtration) or by a discretely delayed filtration.

In the following, we are particularly interested in the delayed information case. Let

$$
\mathcal{F}_{t}^{D}= \begin{cases}\mathcal{F}_{0} & \text { if } t \leq \delta(t), \\ \mathcal{F}_{t-\delta(t)} & \text { if } t>\delta(t),\end{cases}
$$

where $\delta(t)$ is some function on $t$. The above formulation covers the constant delay time model where $\delta(t)=\delta$ (see [4], [12]) and the discrete observation model where $\delta(t)=$ $t-t_{i}^{(m)}, t_{i}^{(m)} \leq t<t_{i+1}^{(m)}$ where $0=t_{0}^{(m)}<t_{1}^{(m)}<\cdots<t_{m}^{(m)}=T$ are the only discrete dates on which the $\left(\mathcal{F}_{t}\right)_{t \geq 0}$ information may be renewed (release dates of the accounting reports of the firm for example, see [6], [16]). In this case, the information of the investors is represented by the progressive enlargement of filtration of $\mathbb{F}^{D}$ with $\tau$ :

$$
\mathcal{G}_{t}^{D}:=\mathcal{F}_{t}^{D} \vee \mathcal{D}_{t}
$$

We call the information related to the filtration $\mathbb{G}^{D}=\left(\mathcal{G}_{t}^{D}\right)_{t \geq 0}$ the "delayed information".

\section{Default probabilities with information asymmetry}

Our aim is to compute the conditional probabilities of default with respect to the different filtrations introduced in the previous section. More precisely, we compute $\mathbb{P}\left(\tau>\theta \mid \mathcal{H}_{t}\right)$ for all $t<\theta$, where the filtration $\left(\mathcal{H}_{t}\right)_{t \geq 0}$ describes the accessible information for the investors. Remark that the default time $\tau$ is a $\left(\mathcal{H}_{t}\right)_{t \geq 0}$ stopping time for all the four levels of information we consider.

\subsection{Full information}

Proposition 3.1 If $\mathcal{H}_{t}=\mathcal{G}_{t}^{M}$ is the full information, then under Assumption 2.1, we have for any $\theta>t$,

$$
\mathbb{P}\left(\tau>\theta \mid \mathcal{G}_{t}^{M}\right)=\frac{1}{p_{t}(L)}\left[E_{\mathbb{P}}\left(p_{\theta}(x) 1_{X_{\theta}^{*}>x} \mid \mathcal{F}_{t}\right)\right]_{x=L}
$$


where $p_{t}(x)(\omega)=\frac{d P_{t}^{L}}{d P^{L}}(\omega, x), P_{t}^{L}(\omega, d x)$ being a regular version of the conditional law of $L$ given $\mathcal{F}_{t}$ and $P^{L}$ being the law of $L$.

Proof: Using the facts that $\mathcal{F}_{\theta}$ and $\sigma(L)$ are independent under $\mathbb{Q}^{L}$, that $E_{\mathbb{Q}^{L}}\left[\frac{d \mathbb{P}}{d \mathbb{Q}^{L}} \mid \mathcal{G}_{t}^{M}\right]=$ $p_{t}(L)$, and that $\mathbb{Q}^{L}$ is identical to $\mathbb{P}$ on $\mathcal{F}_{\infty}$, we have

$$
\begin{aligned}
\mathbb{P}\left(\tau>\theta \mid \mathcal{G}_{t}^{M}\right) & =E_{\mathbb{P}}\left(1_{X_{\theta}^{*}>L} \mid \mathcal{F}_{t} \vee \sigma(L)\right) \\
& =\frac{1}{p_{t}(L)} E_{\mathbb{Q}^{L}}\left(p_{\theta}(L) 1_{X_{\theta}^{*}>L} \mid \mathcal{F}_{t} \vee \sigma(L)\right) \\
& =\frac{1}{p_{t}(L)}\left[E_{\mathbb{Q}^{L}}\left(p_{\theta}(x) 1_{X_{\theta}^{*}>x} \mid \mathcal{F}_{t}\right)\right]_{x=L} \\
& =\frac{1}{p_{t}(L)}\left[E_{\mathbb{P}}\left(p_{\theta}(x) 1_{X_{\theta}^{*}>x} \mid \mathcal{F}_{t}\right)\right]_{x=L}
\end{aligned}
$$

Remark: If $\mathcal{F}_{\theta}$ and $\sigma(L)$ are independent under $\mathbb{P}$, we obtain the simple formula

$$
\mathbb{P}\left(\tau>\theta \mid \mathcal{G}_{t}^{M}\right)=P_{t}^{X_{\theta}^{*}}(] L,+\infty[),
$$

where $P_{t}^{X_{\theta}^{*}}(d y)$ is the regular conditional probability of $X_{\theta}^{*}$ given $\mathcal{F}_{t}$.

\subsection{Progressive information and the delayed case}

The case with progressive information corresponds to the standard reduced form modelling approach and the computation results are well known in the literature (e.g.[17, 8, 1]). In this case, the investor knows the information on the underlying process.

Proposition 3.2 If $\mathcal{H}_{t}=\mathcal{G}_{t}=\mathcal{F}_{t} \vee \mathcal{D}_{t}$ is the progressive information, we have for $\theta>t$

$$
\mathbb{P}\left(\tau>\theta \mid \mathcal{G}_{t}\right)=1_{\tau>t} \frac{\mathbb{E}\left(P_{\theta}^{L}\left(X_{\theta}^{*}\right) \mid \mathcal{F}_{t}\right)}{P_{t}^{L}\left(X_{t}^{*}\right)}
$$

Proof: Classical computation in the progressive enlargement leads to

$$
\mathbb{P}\left(\tau>\theta \mid \mathcal{G}_{t}\right)=1_{\tau>t} \frac{\mathbb{P}\left(\tau>\theta \mid \mathcal{F}_{t}\right)}{\mathbb{P}\left(\tau>t \mid \mathcal{F}_{t}\right)}=1_{\tau>t} \frac{\mathbb{E}\left(S_{\theta} \mid \mathcal{F}_{t}\right)}{S_{t}}, \quad \theta>t
$$

where $S_{t}=\mathbb{P}\left(\tau>t \mid \mathcal{F}_{t}\right)$. In our model, $S$ is given explicitly by $S_{t}=\mathbb{P}\left(X_{t}^{*}>L \mid \mathcal{F}_{t}\right)=$ $P_{t}^{L}\left(X_{t}^{*}\right)$ with $P_{t}^{L}$ being the conditional law of $L$ given $\mathcal{F}_{t}$. This gives the result.

In the classical reduced form models such as the Cox process model, the interpretation of the underlying process is different from the one in the model (1.1). Let $\Lambda_{t}=X_{0}-X_{t}^{*}$. The interpretation of this positive and increasing process is the compensator of default in 
the reduced-form models. The default is defined as the first time that the compensator process reaches the independent upper barrier $\widetilde{L}=X_{0}-L$. The process $\Lambda$ can be calibrated from market data and the barrier $\widetilde{L}$ is supposed to follow the unit exponential law. In this case, we recover the well-known formula $\mathbb{P}\left(\tau>\theta \mid \mathcal{F}_{t}\right)=\mathbb{E}\left[e^{-\left(\Lambda_{\theta}-\Lambda_{t}\right)} \mid \mathcal{F}_{t}\right]$ for $\theta>t$. Furthermore, if $\Lambda$ is absolutely continuous w.r.t. the Lebesgue measure, i.e. $\Lambda_{t}=\int_{0}^{t} \lambda_{s} d s$, then the positive process $\lambda$ is called the default intensity. We have that the process $\left(1_{\tau \leq t}-\int_{0}^{t \wedge \tau} \lambda_{s} d s, t \geq 0\right)$ is a $\mathbb{G}$-martingale.

In the case with delayed information, explicit computations have been given for specific delayed information $\mathbb{G}^{D}$ in the literature such as in $[2,4,6,12]$. Here we just give a general computation formula without discussing the details.

Corollary 3.3 If $\mathcal{H}_{t}=\mathcal{G}_{t}^{D}=\mathcal{F}_{t}^{D} \vee \mathcal{D}_{t}$ is the delayed information, we have for $\theta>t$

$$
\mathbb{P}\left(\tau>\theta \mid \mathcal{G}_{t}^{D}\right)=1_{\tau>t} \frac{\mathbb{E}\left(P_{\theta}^{L}\left(X_{\theta}^{*}\right) \mid \mathcal{F}_{t}^{D}\right)}{\mathbb{E}\left(P_{t}^{L}\left(X_{t}^{*}\right) \mid \mathcal{F}_{t}^{D}\right)} .
$$

Proof: Similar as in the progressive information case,

$$
\mathbb{P}\left(\tau>\theta \mid \mathcal{G}_{t}^{D}\right)=1_{\tau>t} \frac{\mathbb{P}\left(\tau>\theta \mid \mathcal{F}_{t}^{D}\right)}{\mathbb{P}\left(\tau>t \mid \mathcal{F}_{t}^{D}\right)}=1_{\tau>t} \frac{\mathbb{E}\left(S_{\theta} \mid \mathcal{F}_{t}^{D}\right)}{\mathbb{E}\left(S_{t} \mid \mathcal{F}_{t}^{D}\right)}=1_{\tau>t} \frac{\mathbb{E}\left(P_{\theta}^{L}\left(X_{\theta}^{*}\right) \mid \mathcal{F}_{t}^{D}\right)}{\mathbb{E}\left(P_{t}^{L}\left(X_{t}^{*}\right) \mid \mathcal{F}_{t}^{D}\right)}
$$

\subsection{Noisy full information}

In this subsection, $\mathcal{H}_{t}=\mathcal{G}_{t}^{I}$. We consider the particular but useful case in finite horizon time $T$ where $L_{t}=L+\epsilon_{t}, \epsilon_{t}=Z_{T-t}, Z$ being a continuous process with independent increments whose marginal has density $q_{t}$ (this example was introduced in Corcuera et al. [5] to study insider's portfolio optimization problems). For example, $\epsilon_{t}=W_{g(T-t)}$ with $W$ an independent Brownian motion, and $g:[0, T] \rightarrow[0,+\infty)$ a strictly increasing bounded function with $g(0)=0$.

Proposition 3.4 We assume that $\mathcal{H}_{t}=\mathcal{G}_{t}^{I}$ is the noisy full information with $L_{t}=L+\epsilon_{t}$, $\epsilon_{t}=Z_{T-t}, Z$ being a continuous process with independent increments whose marginal has density $q_{t}$. Then we have for $\theta>t$,

$$
\mathbb{P}\left(\tau>\theta \mid \mathcal{G}_{t}^{I}\right)=1_{\tau>t} \frac{\int_{\mathbb{R}} \frac{1}{p_{t}(l)} E_{\mathbb{P}}\left(p_{\theta}(l) 1_{X_{\theta}^{*}>l} \mid \mathcal{F}_{t}\right) q_{T-t}\left(L_{t}-l\right) P_{t}^{L}(d l)}{\int_{\mathbb{R}} 1_{X_{t}^{*}>l} q_{T-t}\left(L_{t}-l\right) P_{t}^{L}(d l)}
$$

where $P_{t}^{L}$ is a regular version of the conditional law of $L$ given $\mathcal{F}_{t}$ and $\frac{1}{p_{t}(l)} E_{\mathbb{P}}\left(p_{\theta}(l) 1_{X_{\theta}^{*}>l} \mid \mathcal{F}_{t}\right)$ is the conditional default probability for the full information on the event $\{L=l\}$ (see Proposition 3.1). 
Proof: We recall that $\mathcal{G}_{t}^{I}=\mathcal{F}_{t}^{I} \vee \mathcal{D}_{t}$. A first step is to compute $\mathbb{P}\left(\tau>\theta \mid \mathcal{F}_{t}^{I}\right)$.

Let $A_{\theta} \in \mathcal{F}_{\theta}$ and $h$ be a bounded measurable function. Using the independence of $\mathcal{F}_{\theta \vee t} \vee \sigma(L)$ and $Z$, we have

$$
\begin{aligned}
\mathbb{E}\left(h(L) 1_{A_{\theta}} \mid \mathcal{F}_{t}^{I}\right) & \left.=\mathbb{E}\left(h(L) 1_{A_{\theta}} \mid \mathcal{F}_{t} \vee \sigma\left(L_{t}\right) \vee \sigma\left(\left(\epsilon_{t}-\epsilon_{s}\right), s \leq t\right)\right)\right) \\
& =\mathbb{E}\left(h(L) 1_{A_{\theta}} \mid \mathcal{F}_{t} \vee \sigma\left(L+\epsilon_{t}\right)\right)
\end{aligned}
$$

Let $P_{t}^{L}(d l)$ be the regular conditional probability of $L$ given $\mathcal{F}_{t}$. Then for $C \in \mathcal{B}\left(\mathbb{R}^{2}\right)$,

$$
\mathbb{P}\left(\left(L, L+\epsilon_{t}\right) \in C \mid \mathcal{F}_{t}\right)=\int_{\mathbb{R}^{2}} 1_{C}(l, x) q_{T-t}(x-l) P_{t}^{L}(d l) d x .
$$

Therefore

$$
\mathbb{E}\left(h(L) \mid \mathcal{F}_{t}^{I}\right)=\frac{\int_{\mathbb{R}} h(l) q_{T-t}\left(L_{t}-l\right) P_{t}^{L}(d l)}{\int_{\mathbb{R}} q_{T-t}\left(L_{t}-l\right) P_{t}^{L}(d l)} .
$$

Hence, if $\theta \leq t$ we have

$$
\mathbb{P}\left(\tau>\theta \mid \mathcal{F}_{t}^{I}\right)=\frac{\int_{\mathbb{R}} 1_{X_{\theta}^{*}>l} q_{T-t}\left(L_{t}-l\right) P_{t}^{L}(d l)}{\int_{\mathbb{R}} q_{T-t}\left(L_{t}-l\right) P_{t}^{L}(d l)} .
$$

If $\theta>t$, we use the following successive conditional expectations

$$
\mathbb{P}\left(\tau>\theta \mid \mathcal{F}_{t} \vee \sigma\left(L+\epsilon_{t}\right)\right)=\mathbb{P}\left(\mathbb{P}\left(\tau>\theta \mid \mathcal{F}_{t} \vee \sigma\left(L+\epsilon_{t}\right) \vee \sigma(L)\right) \mid \mathcal{F}_{t} \vee \sigma\left(L+\epsilon_{t}\right)\right) .
$$

Using the fact that $\epsilon$ is independent to $\mathcal{F}_{T} \vee \sigma(L)$, we have

$\mathbb{P}\left(\tau>\theta \mid \mathcal{F}_{t} \vee \sigma\left(L+\epsilon_{t}\right) \vee \sigma(L)\right)=\mathbb{P}\left(X_{\theta}^{*}>L \mid \mathcal{F}_{t} \vee \sigma\left(\epsilon_{t}\right) \vee \sigma(L)\right)=\mathbb{P}\left(X_{\theta}^{*}>L \mid \mathcal{F}_{t} \vee \sigma(L)\right)=: h_{t}(L)$

where $h_{t}(L)=\frac{1}{p_{t}(L)}\left[E_{\mathbb{P}}\left(p_{\theta}(x) 1_{X_{\theta}^{*}>x} \mid \mathcal{F}_{t}\right)\right]_{x=L}$ corresponds to the conditional default probability for the full information. Therefore

$$
\mathbb{P}\left(\tau>\theta \mid \mathcal{F}_{t}^{I}\right)=\frac{\int_{\mathbb{R}} \frac{1}{p_{t}(l)} E_{\mathbb{P}}\left(p_{\theta}(l) 1_{X_{\theta}^{*}>l} \mid \mathcal{F}_{t}\right) q_{T-t}\left(L_{t}-l\right) P_{t}^{L}(d l)}{\int_{\mathbb{R}} q_{T-t}\left(L_{t}-l\right) P_{t}^{L}(d l)} .
$$

The second step to compute $\mathbb{P}\left(\tau>\theta \mid \mathcal{G}_{t}^{I}\right)$ is straightforward using (3.5) and the wellknown relation in the progressive enlargement of filtration

$$
\mathbb{P}\left(\tau>\theta \mid \mathcal{G}_{t}^{I}\right)=1_{\tau>t} \frac{\mathbb{P}\left(\tau>\theta \mid \mathcal{F}_{t}^{I}\right)}{\mathbb{P}\left(\tau>t \mid \mathcal{F}_{t}^{I}\right)} .
$$

Remark: This proof can be extended to other examples in the infinite horizon. For example, let $\epsilon_{t}=W_{g\left(\frac{1}{t+1}\right)}$ with $W$ an independent Brownian motion, and $g:[0,1] \rightarrow$ $[0,+\infty)$ a strictly increasing bounded function with $g(0)=0$. Then $\epsilon_{t}$ is a centered Gaussian process with independent increments. Let $q_{t}$ be the density of $\epsilon_{t}$. We have for $\theta>t$,

$$
\mathbb{P}\left(\tau>\theta \mid \mathcal{G}_{t}^{I}\right)=1_{\tau>t} \frac{\int_{\mathbb{R}} \frac{1}{p_{t}(l)} E_{\mathbb{P}}\left(p_{\theta}(l) 1_{X_{\theta}^{*}>l} \mid \mathcal{F}_{t}\right) q_{t}\left(L_{t}-l\right) P_{t}^{L}(d l)}{\int_{\mathbb{R}} 1_{X_{t}^{*}>l} q_{t}\left(L_{t}-l\right) P_{t}^{L}(d l)} .
$$




\subsection{Credit spread}

An important quantity in the credit risk analysis is the credit spread defined as the instantaneous conditional default probability at time $t$ :

$$
\lambda_{t}=\lim _{\Delta t \rightarrow 0} \frac{1}{\Delta t} \mathbb{P}\left(t<\tau \leq t+\Delta t \mid \mathcal{H}_{t}\right) \quad \text { a.s. }
$$

In the reduced-form approach with the progressive information, it coincides with the default intensity $\lambda^{\mathbb{F}}$ which is the positive $\mathbb{F}$-adapted process such that $\left(1_{\{\tau \leq t\}}-\int_{0}^{t \wedge \tau} \lambda_{s}^{\mathbb{F}} d s, t \geq\right.$ $0)$ is a $\mathbb{G}$-martingale. In the classical structural approach, the credit spread tends to zero and the intensity does not exist since the default time $\tau$ is a predictable $\mathbb{F}$-stopping time. The credit spread for the delayed information, i.e. when $\mathcal{H}_{t}=\mathcal{F}_{t}^{D}$, has been studied in many papers such as $[6,4,16,12]$. In this case, the credit spread is strictly positive for a short term time.

We note that in the full information case where $\mathcal{H}_{t}=\mathcal{G}_{t}^{M}$, we encounter the same situation as in the classical structural model: the credit spread equals to zero since $L$ is $\mathcal{G}_{t}^{M_{-}}$ measurable. For the insider with the noisy full information $\mathcal{G}_{t}^{I}$, Proposition 3.4 implies that the credit spread remains to be zero. Because of the additional information he has on the default barrier, there is no short-term uncertainty on the default for the insider.

\section{Application and numerical illustrations}

We are now ready to give explicit models for the conditional default probabilities in the different settings of information. The direct application will be the pricing of the credit derivatives such as the defaultable bonds. We implement the formulas in order to quantify numerically how the different levels of information impact the estimations of the default probabilities.

In the literature, the default threshold, if random, is generally supposed to be independent of the filtration $\mathbb{F}$ generated by the firm value process. In this case, the $(\mathrm{H})$ hypothesis is satisfied and the computations can be often simplified. In the following, we first consider an independent threshold case. Moreover, we also give an example where the default threshold is correlated to the underlying process $X$.

We consider the standard Black-Scholes model for the asset values process $X$ :

$$
\frac{d X_{t}}{X_{t}}=\mu d t+\sigma d B_{t}, \quad t \geq 0
$$

where $\mu$ and $\sigma$ are real constants and $B$ is an $\mathbb{F}$-Brownian motion. For $t \geq 0$ and $h, l>0$, one has $([1, \mathrm{p} .69])$

$$
\begin{aligned}
\mathbb{E}_{\mathbb{P}}\left(1_{X_{t}^{*}>l}-1_{X_{t+h}^{*}>l} \mid \mathcal{F}_{t}\right) & =1_{X_{t}^{*}>l}\left(\Phi\left(\frac{-Y_{t}^{l}-\nu h}{\sigma \sqrt{h}}\right)+e^{2 \nu \sigma^{-2} Y_{t}} \Phi\left(\frac{-Y_{t}^{l}+\nu h}{\sigma \sqrt{h}}\right)\right) \\
=: & 1_{X_{t}^{*}>l} \Phi_{t, h}(l)
\end{aligned}
$$


where $\Phi$ is the standard Gaussian cumulative distribution function and

$$
Y_{t}^{l}=\nu t+\sigma B_{t}+\ln \frac{X_{0}}{l}, \quad \text { with } \quad \nu=\mu-\frac{1}{2} \sigma^{2} .
$$

\subsection{Case of an independent default threshold}

The following corollary gives the conditional default probabilities in the Black-Scholes model for any independent default threshold.

Corollary 4.1 We assume that the default threshold $L$ is independent of $\mathcal{F}_{T}$. If the asset process $X$ satisfies the Black-Scholes model, then for any $h>0$, we have

- $\mathbb{P}\left(t+h \geq \tau>t \mid \mathcal{G}_{t}^{M}\right)=1_{\tau>t} \Phi_{t, h}(L)$.

- $\mathbb{P}\left(t+h \geq \tau>t \mid \mathcal{G}_{t}^{I}\right)=1_{\tau>t} \frac{\int 1_{X_{t}^{*}>l} \Phi_{t, h}(l) q_{T-t}\left(L_{t}-l\right) P_{t}^{L}(d l)}{\int 1_{X_{t}^{*}>l} q_{T-t}\left(L_{t}-l\right) P_{t}^{L}(d l)}$.

- $\mathbb{P}\left(t+h \geq \tau>t \mid \mathcal{G}_{t}\right)=1_{\tau>t} \frac{\int \Phi_{t, h}(l) P^{L}(d l)}{\int 1_{X_{t}^{*}>l} P^{L}(d l)}$.

- $\mathbb{P}\left(t+h \geq \tau>t \mid \mathcal{G}_{t}^{D}\right)=1_{\tau>t} \frac{\int\left(\Phi_{t-\delta(t), h+\delta(t)}(l)-\Phi_{t-\delta(t), \delta(t)}(l)\right) P^{L}(d l)}{\int\left(1-\Phi_{t-\delta(t), \delta(t)}(l)\right) P^{L}(d l)}$

where $\Phi_{t, h}$ is defined in (4.1) and $\delta(t)$ is the time delay.

We give numerical comparisons of the conditional default probabilities for different information in the following binomial example where $l_{i} \leq l_{s}$ are the two numerical levels of the threshold. Let $0<\alpha<1$ and

$$
L= \begin{cases}l_{i} & \text { with probability } \alpha, \\ l_{s} & \text { with probability } 1-\alpha .\end{cases}
$$

In the simulation, we take the values: $l_{i}=1, l_{s}=3, \alpha=\frac{1}{2}$.

Comments : The probabilities of default for a full or noisy full information are significantly different from the ones for the progressive or the delayed information. More precisely, if $L=l_{i}$, the manager has fixed the lower value for the default threshold and thus the probability of default will be lower for the full information than for the progressive information (see Figure 1), conversely if $L=l_{s}$ (see Figure 2).

In both cases, the estimation of the default probability for the noisy full information is between the estimations of the full and the progressive information. The difference between the probabilities of default is very significant at the beginning and tends to vanish as time $t$ goes to maturity $T$. If $L$ is constant $\left(l_{i}=l_{s}\right)$, the probabilities of default 

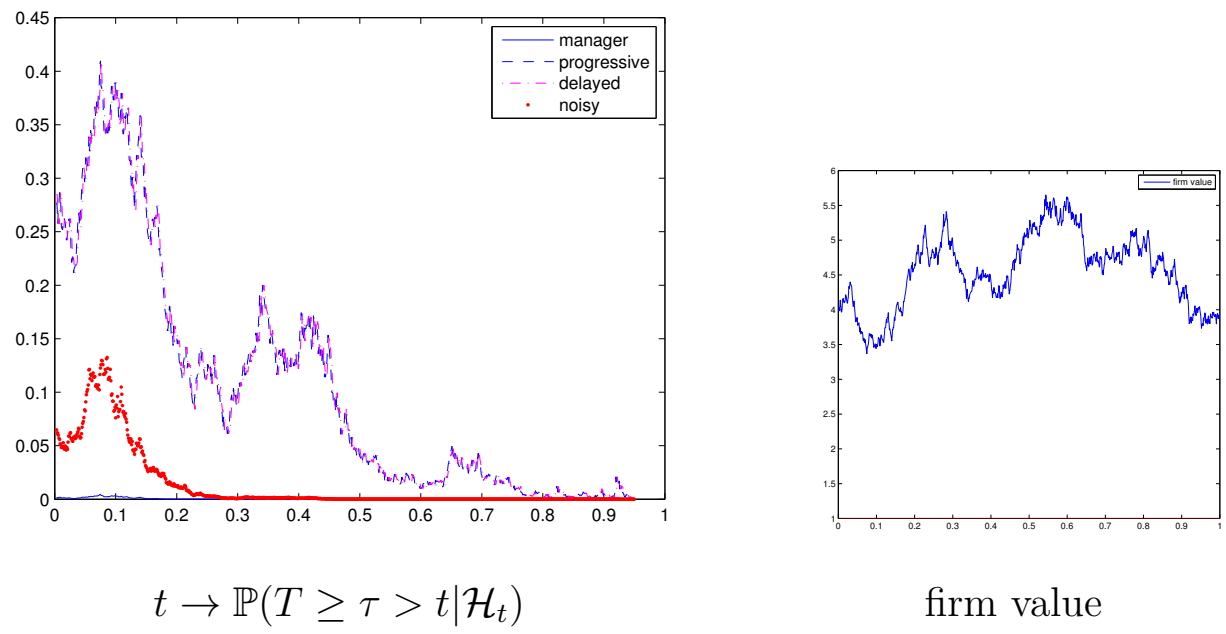

firm value

Figure 1: $L=l_{i}$

are the same, whatever the information we consider (see Figure 3). Not surprisingly, we observe that the variation of the default probabilities is closely related to the variation of the firm value. We note finally that the results between the progressive and the delayed information are very close because we have chosen a small constant delay time.

\subsection{Case of a dependent default threshold}

In practice, the value of the firm or its forecasting play an important role in the manager's decision to fix the default threshold. In the following, we consider the example where

$$
L=l_{i} 1_{[a,+\infty[}\left(X_{A}\right)+l_{s} 1_{[0, a[}\left(X_{A}\right), \quad A>T, \quad l_{i} \leq l_{s} .
$$

The manager chooses the level of $L$ according to a constant threshold $a$ and to the value of the asset process $X$ on some given date $A(A>T$ where $T$ is a fixed horizon time, for example the maturity of the credit derivatives we consider). ${ }^{2}$ If $X_{A} \geq a$, the manager believes the firm on healthy situation and chooses the lower barrier $l_{i}$, otherwise, he chooses the higher barrier to accelerate the default.

We begin by computing the default probability for the managers. By Proposition 3.1, $\mathbb{P}\left(\tau>t+h \mid \mathcal{G}_{t}^{M}\right)=\frac{1}{p_{t}(L)}\left[E_{\mathbb{P}}\left(p_{t+h}(l) 1_{X_{t+h}^{*}>l} \mid \mathcal{F}_{t}\right)\right]_{l=L}$. Compared to the previous independent

\footnotetext{
${ }^{2}$ In this example, the manager knows well the economic situation of the firm so that he has a good prior judgment on whether or not the terminal value of the firm $X_{A}$ will be greater or smaller than the constant threshold $a$.
} 


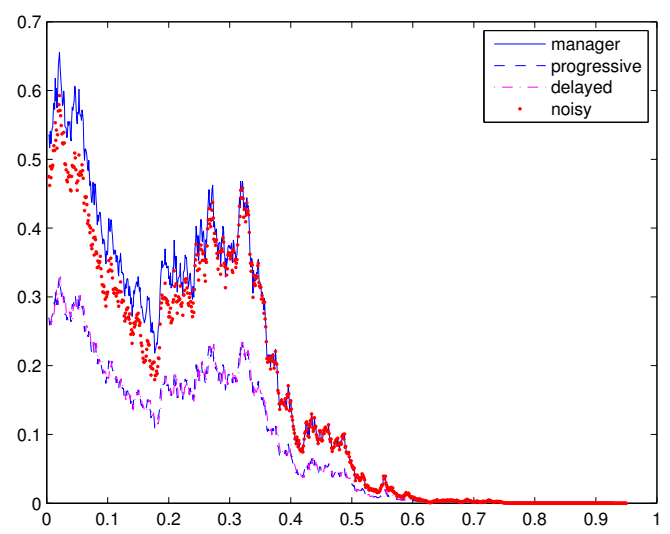

$t \rightarrow \mathbb{P}\left(T \geq \tau>t \mid \mathcal{H}_{t}\right)$

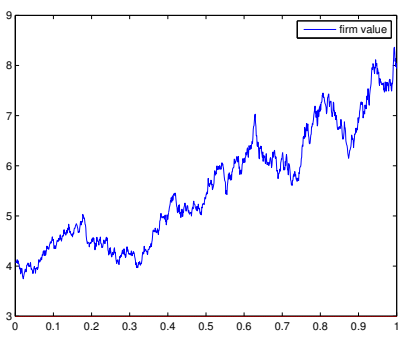

firm value

Figure 2: $L=l_{s}$

case, we first compute the conditional law of $L$ given $\mathcal{F}_{t}$ and then the conditional joint law $\left(p_{t+h}(l), 1_{X_{t+h}^{*}>l}\right)$ given $\mathcal{F}_{t}$.

- We have explicitely for $t<A$

$$
\mathbb{P}\left(L=l_{s} \mid \mathcal{F}_{t}\right)=\mathbb{P}\left(X_{A}<a \mid \mathcal{F}_{t}\right)=\Phi\left(k_{t}\right), \quad \mathbb{P}\left(L=l_{i} \mid \mathcal{F}_{t}\right)=1-\Phi\left(k_{t}\right)
$$

where

$$
k_{t}=\frac{\ln a-\ln X_{0}-\nu A-\sigma B_{t}}{\sigma \sqrt{A-t}} .
$$

Hence

$$
p_{t}\left(l_{s}\right)=\frac{\Phi\left(k_{t}\right)}{\Phi\left(k_{0}\right)}, \quad p_{t}\left(l_{i}\right)=\frac{1-\Phi\left(k_{t}\right)}{1-\Phi\left(k_{0}\right)} .
$$

- Using the following lemma given in [1], we deduce the conditional joint law of $\left(Y_{t+h}^{l}, 1_{X_{t+h}^{*}>l}\right)$ and $\left(p_{t+h}(l), 1_{X_{t+h}^{*}>l}\right)$ given $\mathcal{F}_{t}$.

Lemma 4.2 For $y \geq 0$, on the set $\{\tau>t\}$

$$
\mathbb{P}\left(Y_{t+h}^{l} \geq y, 1_{X_{t+h}^{*}>l} \mid \mathcal{F}_{t}\right)=\Phi\left(\frac{-y+Y_{t}^{l}+\nu h}{\sigma \sqrt{h}}\right)-e^{2 \nu \sigma^{-2} Y_{t}} \Phi\left(\frac{-y-Y_{t}^{l}+\nu h}{\sigma \sqrt{h}}\right)
$$

where $\Phi$ is the standard Gaussian cumulative distribution function, $Y_{t}^{l}=\nu t+\sigma B_{t}+\ln \frac{X_{0}}{l}$ and $\nu=\mu-\frac{1}{2} \sigma^{2}$.

We denote by $f_{t, \theta, l_{s}}(y)$ the conditional density defined by $f_{t, \theta, l_{s}}(y)=\frac{\partial}{\partial y} \mathbb{P}\left(Y_{\theta}^{l_{s}} \geq y, 1_{X_{\theta}^{*}>l_{s}} \mid \mathcal{F}_{t}\right)$.

- Combining these two results, we have for $\theta>t$,

$$
\mathbb{P}\left(\tau>\theta \mid \mathcal{G}_{t}^{M}\right)=1_{L=l_{s}} \frac{1}{\Phi\left(k_{t}\right)} \mathbb{E}\left(\Phi\left(k_{\theta}\right) 1_{X_{\theta}^{*}>l_{s}} \mid \mathcal{F}_{t}\right)+1_{L=l_{i}} \frac{1}{1-\Phi\left(k_{t}\right)} \mathbb{E}\left(\left(1-\Phi\left(k_{\theta}\right)\right) 1_{X_{\theta}^{*}>l_{i}} \mid \mathcal{F}_{t}\right) .
$$




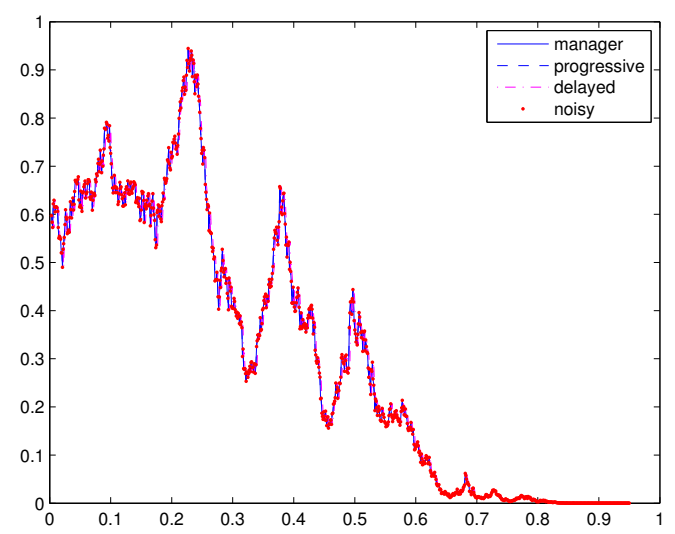

$t \rightarrow \mathbb{P}\left(T \geq \tau>t \mid \mathcal{H}_{t}\right)$

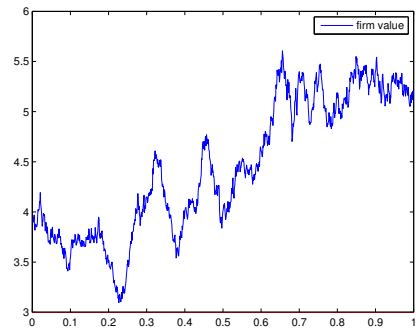

firm value

Figure 3: $l_{i}=l_{s}: L$ constant

where $\Phi\left(k_{t}\right)=g_{l_{s}}\left(Y_{t}^{l_{s}}\right)$ with $g_{l_{s}}(x)=\Phi\left(\frac{\ln \frac{a}{l_{s}}-x-\nu(A-t)}{\sigma \sqrt{A-t}}\right)$ and

$$
\begin{aligned}
\mathbb{E}\left(\Phi\left(k_{\theta}\right) 1_{X_{\theta}^{*}>l_{s}} \mid \mathcal{F}_{t}\right) & =\mathbb{E}\left(g_{l_{s}}\left(Y_{\theta}^{l_{s}}\right) 1_{\left(Y^{\left.l_{s}\right)_{\theta}^{*}>0}\right.} \mid \mathcal{F}_{t}\right)=1_{X_{t}^{*}>l_{s}} \int_{0}^{\infty} g_{l_{s}}(y) f_{t, \theta, l_{s}}(y) d y \\
\mathbb{E}\left(\left(1-\Phi\left(k_{\theta}\right)\right) 1_{X_{\theta}^{*}>l_{i}} \mid \mathcal{F}_{t}\right) & =1_{X_{t}^{*}>l_{i}} \int_{0}^{\infty}\left(1-g_{l_{i}}(y)\right) f_{t, \theta, l_{i}}(y) d y .
\end{aligned}
$$

This gives the conditional default probability for the full information.

The result for the noisy information is then straightforward using Proposition 3.4. The progressive and the delayed case are obtained by classical computations. For the numerical illustrations, we have similar observations to those of the previous section.

\section{Conclusions}

We have investigated the impact of different information levels on the conditional default probabilities. The conditional survival probability plays an important role in the pricing of credit derivatives (we refer the reader to a forthcoming work [13]). For example let us consider a defaultable bond with zero recovery, that is, the buyer of the bond receives 1 euro if there is no default and zero otherwise. Then the price of such a product is exactly the conditional survival probability with respect to the accessible information.

Whereas the information on the value process of the firm has been widely studied, relatively few works concern the information on the default threshold. Our approach combines the initial and the progressive enlargement of filtrations in the modelling of information flows. Our results show that the information on the default threshold also have a significant influence in the credit risk analysis and deserve to be studied in more details. 


\section{References}

[1] Bielecki, T.R., Rutkowski, M., 2002. Credit Risk: Modeling, Valuation and Hedging, Springer-Verlag.

[2] Çetin, U., Jarrow, R., Protter, P., Yıldırım, Y., 2004. Modeling credit risk with partial information, Annals of Applied Probability, 14(3), 1167-1178.

[3] Coculescu, D., Geman, H., Jeanblanc, M., 2006. Valuation of default sensitive claims under imperfect Information, Finance and Stochastics, 12, 195-218.

[4] Collin-Dufresne, P., Goldstein, R., Helwege, J., 2003 Is credit event risk priced? Modelling contagion via the updating of beliefs, preprint.

[5] Corcuera, J.M., Imkeller, P., Kohatsu-Higa, A., Nualart, D., 2004. Additional utility of insiders with imperfect dynamical information, Finance and Stochastics, $8,437-450$.

[6] Duffie, D., Lando, D. 2001. Term structures of credit spreads with incomplete accounting Information, Econometrica, 69, 633-664.

[7] El Karoui, N., 1999. Modélisation de l'information, Lecture Notes, Ecole d'été CEA-EDF-INRIA.

[8] Elliott, R., Jeanblanc, M. and Yor, M., 2000. On models of default risk, Mathematical Finance 10, 179-195.

[9] Giesecke K, 2006. Default and information. Journal of Economic Dynamics and Control, 30, 2281-2303.

[10] Giesecke K, Goldberg L. R., 2008. The market price of credit risk : the impact of asymmetric information. Preprint.

[11] Grorud, A., Pontier, M., 1998. Insider trading in a continuous time market model, International Journal of Theorical and Applied Finance 1, 331-347.

[12] Guo, X., Jarrow, R., Zeng, Y., 2008. Credit risk with incomplete information, to appear in Mathematics of Operations Research.

[13] Hillairet, C., Jiao, Y., 2010. Information asymmetry in pricing of credit derivatives, to appear in International Journal of Theorical and Applied Finance.

[14] Jacod, J., 1979. Calcul stochastique et problèmes de martingales, Lecture Notes 714, Springer-Verlag, New York.

[15] Jacod, J., 1985. Grossissement initial, hypothèse (H') et théorème de Girsanov, Lecture Notes 1118, Springer-Verlag, 15-35. 
[16] Jeanblanc, M., Valchev, S., 2005. Partial information and hazard process, International Journal of Theoretical and Applied Finance, 8(6), 807-838.

[17] Jeulin, J., Yor, M., 1978. Grossissement d'une filtration et semi-martingales: formules explicites, Séminaire de Probabilités (Strasbourg), 12, 78-97, SpringerVerlag.

[18] Lando, D., 1998. On Cox processes and credit risky securities, Review of Derivatives Research, 2, 99-120.

[19] Leland, H., 1994. Corporate debt value, bond convenants and optimal capital structure, Journal of Finance 49(4), 1213-1252.

[20] Merton, R., 1974. On the pricing of corporate debt: the risk structure of interest rates, Journal of Finance 29, 449-470. 